\title{
The Effects of Posterior Spinal Fusion in Patients with Limitations of Anterior Approach: The Experience of Six Cases
}

\author{
Hojjat Hossein Pourfeizi ${ }^{1}$, Ali Sadighi ${ }^{2}$, Alireza Sadeghpour ${ }^{1}$, Bahamin Attar ${ }^{3 *}$, Zahra Azizian ${ }^{4}$ \\ ${ }^{1}$ Associate Professor of Orthopedic Surgery, Department of Orthopedics Surgery, Faculty of Medicine, Shohada Hospital, Tabriz University of Medical
Sciences, Tabriz, Iran
${ }^{2}$ Professor of Orthopedic Surgery, Department of Orthopedics Surgery, Faculty of Medicine, Shohada Hospital, Tabriz University of Medical Sciences,
Tabriz, Iran
${ }^{3}$ Orthopedic Surgery Resident, Department of Orthopedics Surgery, Faculty of Medicine, Shohada Hospital, Tabriz University of Medical Sciences, Tabriz,
Iran
${ }^{4}$ Dermatology Resident, Rasoul-e-Akram Hospital, Iran University of Medical Sciences, Tehran, Iran
}

*Corresponding Author Address: Orthopedic Department, Shohada Hospital, Tabriz University of Medical Sciences, Tabriz, Iran. Tel:+989121998184. Fax:+984133340830. E-mail:tdoc4146@yahoo.com

\section{Abstract}

Background \& Importance: Spinal deformity is one of the most common disorders among the vertebral diseases that results from unnatural curvatures of spine, such as scoliosis and kyphosis. Spinal fusion surgery is used most commonly to treat certain types of spinal deformity. Herein, we reported the efficacy of posterior spinal fusion accompanied by instrumentation in patients with the limitations of the anterior approach.

Case Presentation: This case series study included six patients. There were three cases of severe gibbus deformities, a case of kyphosis deformity and two cases of both kyphosis and scoliosis. Two cases of gibbus deformities had undergone spinal fusion with instrumentation, and relatively satisfactory correction was observed for both patients. One patient, who had been treated with posterior decompression and fixation, returned to leading his normal active life. One case of severe deformities had no dynamic problems four years after receiving the instrumentation surgery.

Conclusion: The outcomes of posterior spinal fusion surgery were found to be satisfactory in patients with the limitations of the anterior approach.

Keywords: : Spinal Deformity; Spinal Fusion; Anterior Approach

Please cite this paper as: Hossein Pourfeizi H, Sadighi A, Sadeghpour A, Attar B, Azizian Z. The Effects of Posterior Spinal Fusion in Patients with Limitations of Anterior Approach: The Experience of Six Cases. Iran. J. Neurosurg. 2016;2(3):15-18

\section{Background \& Importance}

One of the main causes of neurological signs and symptoms, especially in lower extremities are vertebral column deformities [1]. Kyphotic deformities are more common than scoliosis among diseases leading to neurological problems $[1,2]$. According to our experiences, the upper thoracic kyphosis and scoliosis, which are not so cosmetically exposed [3-5] may cause sign and symptoms. Best treatment for these patients is classical approach, anterior decompression and anterior-posterior fixation [6]. But studies show that in elderly patients or those with apex deformity of thoracic region, the anterior approach may lead to considerable adverse effects [7]. In this paper, we reported the efficacy of immobilization and fusion accompanied by instrumentation in cases with anterior approaches limitation. The goals of surgery were to correct the curvature and restore the function with minimum complications in patients with the limitation of anterior approach.

\section{Case Presentation}

\section{Case 1}

A 46-year-old woman admitted for lower extremities dynamic weakness and disability for independent walking for 8 months. She had history of gibbus deformity in childhood. Also she experienced mostly mechanical stimulated back pain since 6 years ago leading to her current disability. Neurological examination showed muscle force 2-3/5, lower extremity DRTs: 4+, positive Babinski reflex and she admitted to sphincter malfunction occasionally. In plain lateral radiography, the gibbus deformity of thoracolumbar region was visible and in plain anteroposterior radiograph no scoliosis was seen. In MRI, there were no space occupying mass in spinal canal and cord protraction was obvious in apex region of kyphosis. As the patient had overweight with chronic deformity of vertebral column, the risk of anterior approach intervention was high. So patient underwent posterior spinal fusion with no effort of deformity correction [because of the rigidity] and instrumentation performed, using pedicle screw in thoracic and lumbar region. Patient discharged 10 days after operation. The operation led to stoppage of progression in weakness. After 8 months muscle forces were improved and she could walk independently. Her sphincter's function normalized, and now it has been passed three years since her operation.

\section{Case 2}

A 70-year-old woman admitted for inability to walk for two months. The dynamical weakness started from 1 year ago and progressed, as she could not walk independently. She had positive SLR sign and had some sphincter malfunction of periodic urine retention. In physical examination, there was a gibbus deformity in thoracolumbar region of vertebral column [from childhood]. Complete blockage was obvious in apex of gibbus in MRI. Because of patient's aging and higher possibility of side effects for anterior approach, which demanded operation of retroperitoneal thorax and ligature of segmental vessels in kyphosis apex and subsequent cord ischemia, posterior approach was used only. The operation included posterior fixation and fusion using thoracic 
and lumbar pedicle screw and in situ fusion with no effort of deformity correction. Oneweek afteroperation, lower extremity force which was $2 / 5$, get weaker and ended complete disability and sphincter malfunction turned into complete dysfunction. Physiotherapy treatment started to prevent muscle atrophy and fix lower extremity joints' limitation of motion. After 2 months, foot fingers motor function improved gradually and within 8 months patient became independent with her moves and routines. It has been passed 2 years since operation.

\section{Case 3}

A 68-year-old woman was admitted for inability to walk since one month ago. In physical examination, muscle forces were $2 / 5$ and $3 / 5$ in left and right lower extremities respectively with increased deep tendon reflexes (DTRs) and positive Babinski signs. Plain radiography showed kyphoscoliosis of thoracic region and myelogram blockage in MRI was obvious. Given that the patient was old aged, our choice was posterior spinal fusion procedure. The operation was carried out through opening the apex of deformity in spinal cord and decompression by removing lamina and pedicle in concave side of deformity, and this led to release spinal cord off the pressure and to fix by pedicle-screw fusion. Two weeks after operation, the patient was hospitalized in ICU with chief complaint of dyspnea and clinical symptoms of pulmonary thromboembolism. During two months, she had dynamic improvements, and she was able to walk by means of walker aid and did her routines independently. It has been four years since her operation, and she can walk by means of a stick and she is independent with her routines. She complains of her lower extremities pain which is controlled by analgesics.

\section{Case 4}

A 50-year-old woman able to walk and do her routines independently was admitted for fatigue in lower extremities. In physical examination, there was an increase in DTRs of lower extremity, and both the right and left leg muscle force were $4 / 5$. Other examinations revealed chronic gibbus in lower thoracic region. Because of high risk of anterior approach for the patient, we preferred to have in situ fixation and fusion with no effort to reform the deformity. After surgery, the lower extremities movement faced some limit in recovery room, but after $24 \mathrm{~h}$ the limitation vanished. After one week , she was discharged. Gradually, patient showed problems in lower extremity movement, and finally it caused complete paralysis. Two months after operation when there was no improvement in signs and symptoms, the patient showed dyspnea and passed away suddenly, probably because of pulmonary thromboembolism.

\section{Case 5}

A 16-year-old boy was admitted due to exhaustion of lower extremities for few months. Gradually, gait disorder was revealed which caused complete disability. In clinical examination, right leg was immobile [muscle force 1/5] and muscle force of left foot was $2 / 5$ and sensory level of T4 was present. The patient had no sphincter malfunction but he had bilateral Babinski. Upper extremities had no dynamic or sensory dysfunction. In plain radiography, 65-degree scoliosis (convexity to the left side) with T4 apex and local 70-degree kyphosis were obvious (Figure 1).

In cord, compression with decrease in diameter was obvious in CT myelography. Diameter in traumatic region was less than other places. In an axial view of CT myelography, cord was compressed in concave site. The patient underwent posterior spinal fusion (PSF) surgery with relative fixation of deformity. Gradually, patient's motion got better, and he could walk three months after the surgery. He was completely fine and capable of running his own routine at one year after the operation (Figure 2).

\section{Case 6}

A 14-year-old boy was admitted for fatigue and frequent fall. In clinical examination, kypho-scoliosis of upper thoracic region was diagnosed. In neurological tests, sensory level in T4 was detected, and motor force of both right and left lower extremities were $3 / 5$ with no sphincter dysfunction. In stagnara view of radiography, 100-degree scoliosis [convexity to the left] and 50-degree local kyphosis were visible. In CT myelography, cord compression in concave side of apical vertebrae was visible. The patient underwent PSF surgery. Because of severity of deformity, bilateral fixation was impossible, and fixations of concave side were done as well as posterior spinal fusion and decortication using allograft. Neurological signs and symptoms were improved in few months and in the last visit. The patient had no dynamic problem during four years after surgery. It has passed 12 years since surgery.

\section{Discussion}

We observed that PSF accompanied by instrumentation could be an appropriate alternative to anterior approach in some patients with limitations. Previous studies suggest combined anterior and posterior fusion approaches to correct thoracolumbar spine deformities because this method seems to be more efficient and impressive on neurological complications despite its life threatening risks [8,9]. Although empowered studies have not been conducted to compare the efficacy of these two methods, the evidence acts in the favor of combined method [10-12]; however, our findings showed different outcomes. In three cases with severe gibbus deformities, we only had fusion and instrumentation, and it worked for two cases, though one of the patients died of thromboembolism (case 2). In the case cured by posterior decompression and fixation, the patient got well and was able to run the routine life. In one case (case 6), instrumentation was used as a result of severe deformity and kyphosis and scoliosis in upper thoracic region concavity. In spite of above-mentioned considerations, this study had not enough competence to abolish one method and prove another one, and clinical trials are recommended to reach verified conclusion.

\section{Funding}

None.

\section{Conflicts of Interest}

The authors declare that they have no conflicts of interest.

\section{Authors' Contribution}

Conception and Design: Bahamin Attar, Data Collection: Bahamin Attar, Drafting the Article: Hojjat Hossein Pourfeizi, Critical Revising the Article: Zahra Azizian, Reviewed Submitted Version of Manuscript: Alireza Sadeghpour, Approved the Final Version of the Manuscript: Ali Sadighi

\section{References}

1. Good CR, Auerbach JD, O'Leary PT, Schuler TC. Adult Spine Deformity. Current Reviews in Musculoskeletal Medicine. 2011;4(4):159-67.

2. Youssef J, Orndorff D, Patty C, Scott M, Price H, Hamlin L, et al. Current status of adult spinal deformity. Global Spine Journal. 2013;3(01):051-62. 

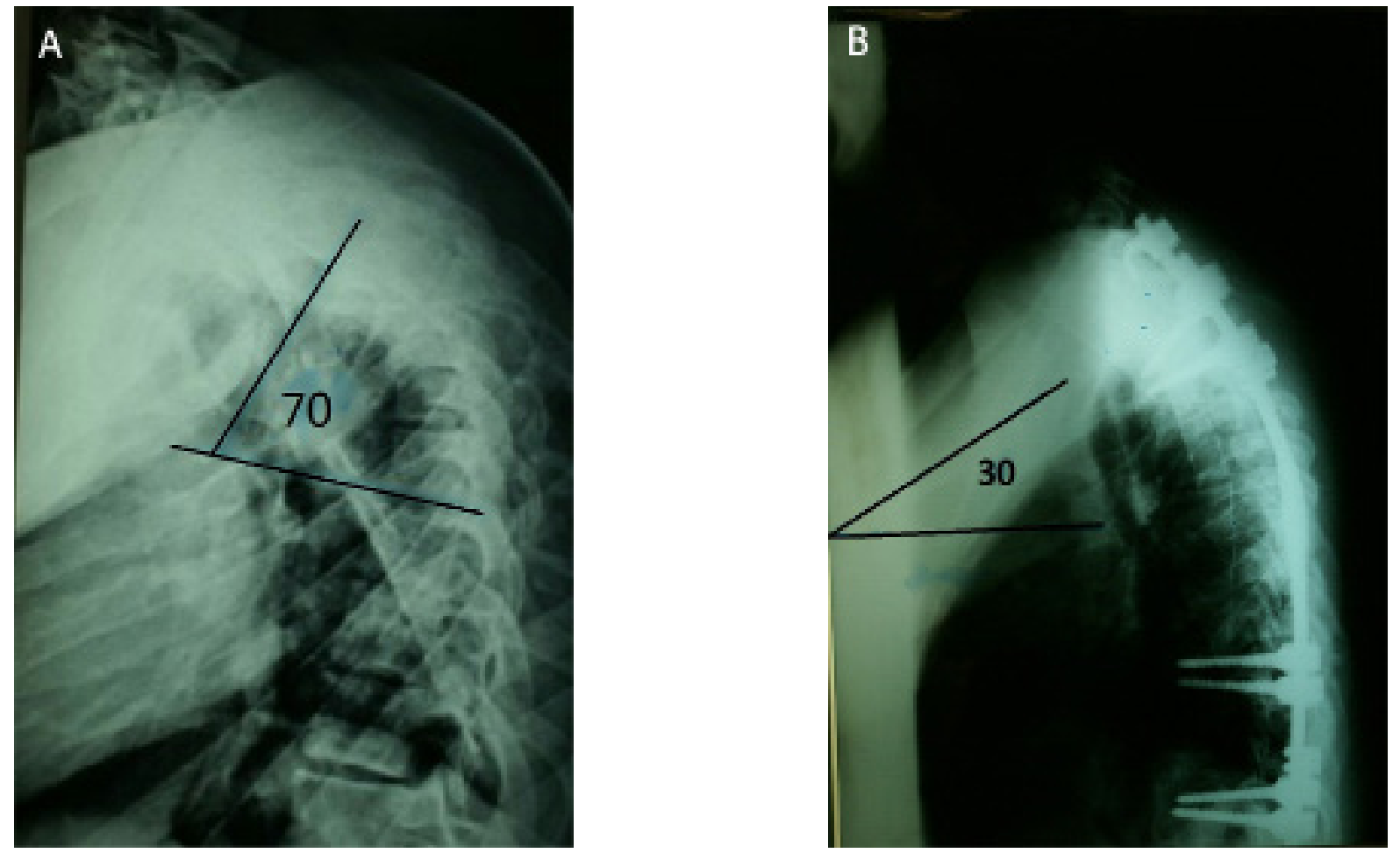

Figure 1. Plain Radiographs Lateral View Demonstrating a Kyphotic Angle of: (A) 70 Degrees Preoperatively, (B) 30 Degrees Postoperatively
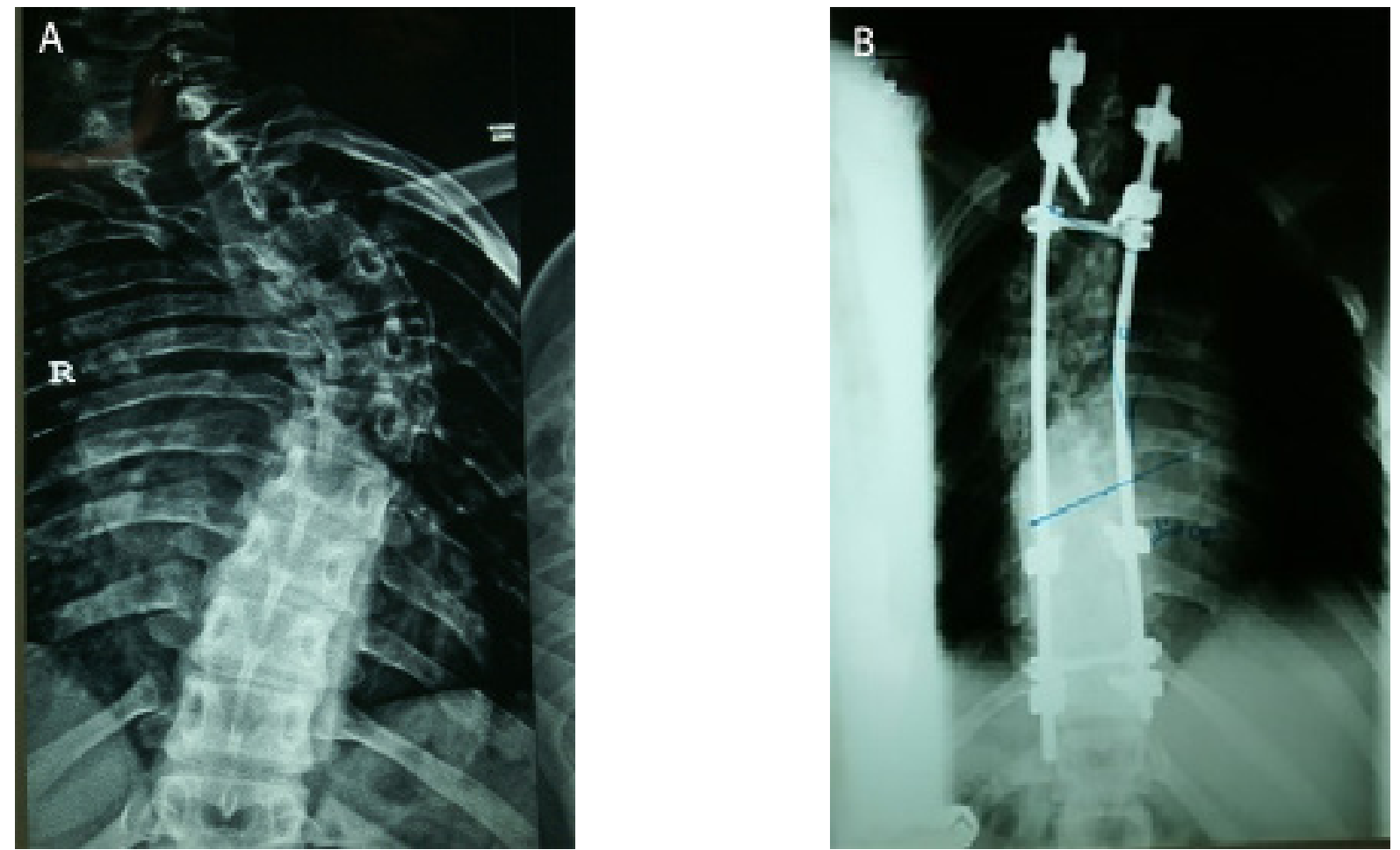

Figure 2. Plain Radiographs Anteroposterior View: (A) Preoperative Plain Showing 65 Degrees Scoliosis, (B) 12 Months Postoperative Plain Showing the Correct Alignment of the Spine

3. Debnath UK, Goel V, Harshavardhana N, Webb JK. Congenital scoliosis-Quo vadis? Indian Journal of Orthopaedics. 2010;44(2):137.

4. Katzman WB, Wanek L, Shepherd JA, Sellmeyer DE. Age-related hyperkyphosis: its causes, consequences, and management. Journal of Orthopaedic \& Sports Physical Therapy. 2010;40(6):352-60.

5. Janicki JA, Alman B. Scoliosis: Review of diagnosis and treatment. Paediatrics \& Child Health. 2007;12(9):771.

6. Mak KC, Cheung KM. Surgical treatment of acute TB spondylitis: indications and outcomes. European Spine Journal. 2013;22(4):603-11.
7. Yau A, Hsu L, O'BRIEN J, Hodgson A. Tuberculous kyphosis. Journal of Bone \& Joint Surgery American version. 1974;56(7):1419-34.

8. Moon M-S, Kim S-S, Lee B-J, Moon J-L, Moon Y-W. Surgical management of severe rigid tuberculous kyphosis of dorsolumbar spine. International Orthopaedics. 2011;35(1):75-81.

9. Wang Y, Lenke LG. Vertebral column decancellation for the management of sharp angular spinal deformity. European Spine Journal. 2011;20(10):1703-10.

10. Chopko BW. Percutaneous thoracolumbar decompression combined with percutaneous pedicle screw fixation and fusion: a method for treating spinal 
degenerative pain in a biplane angiography suite with the avoidance of general anesthesia. Journal of Spine Surgery. 2016;2(2):122.

11. Pourfeizi HH, Sales JG, Tabrizi A, Borran G, Alavi S. Comparison of the combined anterior-posterior approach versus posterior-only approach in scoliosis treatment. Asian Spine Journal. 2014;8(1):8-12.

12. Pflugmacher R, Agarwal A, Kandziora F, Cyrus K. Balloon kyphoplasty combined with posterior instrumentation for the treatment of burst fractures of the spine-1-year results. Journal of Orthopaedic Trauma. 2009;23(2):126-31.

\section{Comments}

The authors presented a case series of various forms of spinal deformity operated just by laminectomy and posterior pedicular screw insertion and fusion. Because the imaging of the cases were not shown completely, the basis of their decision making was not clear. The degree of correction postoperatively was not mentioned. In addition, it was not mentioned why some patients considered high-risk and just posterior method was applied for them. The cause of severe paraparesis in case 5 was not clear.

Generally, if the goal of the surgery is only decompression without deformity correction, adequate decompression could be achieved with laminectomy and in-situ fusion, but if the deformity itself also causes compression, the deformity correction should be considered. With development of techniques in deformity surgery, acceptable correction can be achieved only via posterior method and the need for anterior method becomes more and more limited.

In these series, 2 out of 6 cases (about 33\%) experienced worsening of neurologic condition, which indicates that the laminectomy without deformity correction is not sufficient alone and even may cause further damage. The spinal deformity surgery requires complete evaluation of the case and the ultimate goal of the surgery is very important in method selection.

Amir Azarhomayoun, MD,

1. Sina Trauma and Surgery Research Center, Tehran University of Medical

Sciences, (TUMS), Tehran, Iran

2. Gundishapour Academy of Neuroscience (GAN), Ahvaz, Iran 